\title{
From cell senescence to age-related diseases: differential mechanisms of action of senescence-associated secretory phenotypes
}

\author{
Hae-Ok Byun ${ }^{1,2}$, Young-Kyoung Lee ${ }^{1,2}$, Jeong-Min Kim ${ }^{1,3}$ E Gyesoon Yoon ${ }^{1,2, *}$ \\ ${ }^{1}$ Department of Biochemistry, Ajou University School of Medicine, ${ }^{2}$ Department of Biomedical Science, Graduate School, ${ }^{3}$ College of \\ Natural Sciences, Ajou University, Suwon 16499, Korea
}

Cellular senescence is a process by which cells enter a state of permanent cell cycle arrest. It is commonly believed to underlie organismal aging and age-associated diseases. However, the mechanism by which cellular senescence contributes to aging and age-associated pathologies remains unclear. Recent studies showed that senescent cells exert detrimental effects on the tissue microenvironment, generating pathological facilitators or aggravators. The most significant environmental effector resulting from senescent cells is the senescence-associated secretory phenotype (SASP), which is constituted by a strikingly increased expression and secretion of diverse pro-inflammatory cytokines. Careful investigation into the components of SASPs and their mechanism of action, may improve our understanding of the pathological backgrounds of age-associated diseases. In this review, we focus on the differential expression of SASP-related genes, in addition to SASP components, during the progress of senescence. We also provide a perspective on the possible action mechanisms of SASP components, and potential contributions of SASP-expressing senescent cells, to age-associated pathologies. [BMB Reports 2015; 48(10): 549558]

\section{INTRODUCTION}

Cellular senescence is causally implicated in biological aging; there is evidence of accumulated senescent cells in tissues of chronologically aging organisms (1-3). In addition, potential links between cellular senescence and age-related diseases, including osteoarthritis (4) and atherosclerosis (5), Parkinson's disease (6), and cancer (7), have been reported and are supported

*Corresponding author. Tel: +82-31-219-5054; Fax: +82-31-2195059; E-mail: ypeace@ajou.ac.kr

http://dx.doi.org/10.5483/BMBRep.2015.48.10.122

Received 23 June 2015

Keywords: Age-associated diseases, Cell senescence, Differential expression, Senescence-associated secretory phenotypes (SASP) by a recent report that removal of senescent cells can prevent or delay tissue dysfunction and extend the "healthspan" (8).

Cellular senescence refers to a permanently arrested state of cell growth that displays unresponsiveness to growth factors. It was originally described in terms of exhaustion of the replicative capacity of cultured primary fibroblasts (2) and was thought to be due in part to telomere attrition (the gradual loss of DNA at the ends of chromosomes that accompanies continuous cell division) $(9,10)$. It has been shown that telomere attrition generates a persistent DNA damage response, which initiates and maintains senescent growth arrest (10-12). However, cell senescence can also be induced by various stresses, including oncogenic activation, UV radiation, and chemical damage or therapeutic drug toxicity (13-15). The often unspecified causes and unclear molecular mechanisms of senescence provokes one of the fundamental questions in the biology of aging and clinical geriatrics: how does cellular senescence contribute to age-related diseases?

Despite the different causes of senescence, the senescent cells display several prominent common phenotypes, in addition to irreversible growth arrest and unresponsiveness to growth factors. Major representative phenotypes are an enlarged and flat cellular morphology with intracellular mass increase (16); increased reactive oxygen species (ROS) production and accumulation of consequent ROS-mediated damage products $(13,17)$; acquisition of senescence-associated $\beta$-galactosidase activity (SA- $\beta$-gal) (18); a discernible change in chromatin organization known as senescence-associated heterochromatin foci formation (SAHF) $(19,20)$; and acquisition of the senescence-associated secretory phenotype (SASP), which includes secreted inflammatory, growth-regulating, and tissue-remodeling factors $(21,22)$. Although these phenotypic changes are often used as indications for cellular senescence, their association to each other and their exact relevance to senescence itself, organismal aging, and age-related diseases, are not clearly understood. One established senescent feature linked to agerelated pathologies is the fact that senescent cells lose their proliferation capacity and consequent normal cell turnover, thereby weakening tissue repair and regeneration, and leading to decrements in function (23). Another feature recently draw-

ISSN: 1976-670X (electronic edition)

Copyright (C) 2015 by the The Korean Society for Biochemistry and Molecular Biology

(c) This is an open-access article distributed under the terms of the Creative Commons Attribution Non-Commercial License (http://creativecommons.org/licenses/by-nc/4.0) which permits unrestricted non-commercial use, distribution, and reproduction in any medium, provided the original work is properly cited. 
ing attention is the release of SASPs which, due to their potent autocrine and paracrine activities, control the functions and activities of the surrounding cells and also modify the extracellular microenvironments $(21,22)$. However, senescent cells express and release a variety of SASP components, with different levels of individual SASP components at different stages of senescent progress (24); thus, it is quite difficult to unravel the link between individual and combined SASP components and age-related pathologies.

Before discussing the action modes of SASP components, several salient features need to be mentioned. First, there are many types of SASP components, such as cytokines, chemokines, growth factors, and proteases, which are expressed and involved in senescence at different individual levels. This indicates that the overall contribution to a specific pathogenesis of the combined actions of all SASP components may be quite different from the known effects of individual SASP components. Second, the senescent cells also express and release regulatory or inhibitory factors, such as tissue inhibitors of metalloproteinases (TIMPs), plasminogen activator inhibitors (PAI), and insulin-like growth factor-binding proteins (IGFBPs). This implies that the ultimate activity of a single SASP component may not be determined only by the number of SASP components involved, but also by the combinatorial action of the SASP components along with their regulatory factors. Third, the progress of senescence alters the expression levels of SASP receptors, modifying cellular reactivity to specific SASP ligands. Fourth, the types and levels of SASP components vary among the stages of senescence progression, suggesting that senescent cells at different stages may affect the age-related pathogenesis differently. Therefore, careful consideration of the altered expression profiles of individual SASP components and their associated regulatory factors and receptors may allow us to hypothesize the possible mechanism of action by which individual SASP components modulate the surrounding tissue microenvironment, and to determine how senescent cells communicate with the surrounding cells.

In this review, we discuss various studies relating to the secretion of SASP components from senescent cells and their involvement in age-related pathologies. Here, the aforementioned features of SASP components and their associated gene products are collectively referred to as SASP-related factors. We also consider the possible action mechanisms of individual SASP components on the tissue microenvironment, and finally propose the potential contributions of senescent cells to senescence-related pathologies.

\section{SPECIFIC VERSUS OVERALL GENE EXPRESSION CHANGES IN SENESCENCE}

Cell size is fairly homogeneous during the early passages of human fetal fibroblasts, while cell size at the terminal stages (senescence) of the in vitro cellular life span is large and heterogeneous $(25,26)$. Similarly, increases in cell size (surface area) and cell mass (components) are generally seen in most cases of cellular senescence triggered by various stresses. Thus, this enlarged cell morphology and size is the most prominent senescent phenotype, which allows us to judge, by appearance, whether or not cells are progressing to senescence. How, then, do senescent cells acquire this phenotype? The enlarged cell size reflects an increase in cell mass, generally in terms of both molecular and organellar components (27-29). However, it is unclear whether the increase in mass is confined to specific cellular molecules and compartments, or whether it is caused by a random and uncontrolled increase.

The majority of senescent cells are in the $G_{1}$ phase of the cell cycle (i.e., $G_{1}, S, G_{2}$, and $M$ phase, respectively), with an overall delay seen in cell cycle progression, indicating that the $\mathrm{G}_{1}$ checkpoints are critical controls for senescence $(30,31)$. This stable G1 arrest is mainly executed by an interplay between the Rb and p53 tumor suppressor pathways $(11,32)$. Senescent cells express activated p53 transcription factor (33, 34) and, consequently, elevated levels of p21 $1^{\text {Cip1/Waf1 }}$ (35), p1 $5^{\text {INK4b }}(36,37)$, and p16 ${ }^{\text {INK4a }}(38)$; also, they are unable to hyperphosphorylate $\mathrm{Rb}$ protein in response to mitogenic stimulation (39). Activation of these cell cycle checkpoints comprises an important mechanism of cell cycle arrest in senescent cells (40). However, $G_{1}$ is the phase at which the cell grows in size by synthesizing the mRNA and proteins required for cell components, as well as some specific proteins required for DNA synthesis. Once the required cell growth and mass increase has taken place, the cell enters the next phase of the cell cycle, the S phase. Senescent cells in G1 arrest, which is the result of an inability to transition from $\mathrm{G} 1$ to $S$ without the cessation of synthesis of cellular molecules and components, may result in enlarged cell morphology. Ultimately, this representative senescent phenotype, i.e. progressive enlargement of cell morphology, is tightly linked with another well-known senescent feature: senescent cells remain metabolically active, which includes an overall increase in gene expression (41), despite the loss of their replicative capacity.

Enhanced protein synthesis during senescent arrest is maintained by mTOR activation and upregulated activity of phosphatidylinositol 3-kinase (PI3K), an upstream activator of mTOR (42-45). Moreover, GSK3-mediated augmentation of lipogenesis and glycogenesis has been reported to be critically linked with an increase in the overall mass of organelles (such as mitochondria, lysosome, Golgi, and ER) and cell granularity (46, 47). In particular, increased mass of lysosomes and mitochondria has been observed in both senescent cells and aged tissues $(29,48,49)$. The combined activity of augmented lipogenesis and protein synthesis leads to the increase in organellar formation. However, the imbalance between anabolic activities, including protein synthesis and organellar biogenesis, and cell cycle progression contributes to the abnormal cell volume increase.

Alongside the overall increased synthesis of mRNAs and proteins, the senescent cells also have an extremely altered ex- 
pression of specific genes, that are often referred to as senescence-associated genes. These include p53 (33, 34), $\mathrm{p} 21^{\text {Cip } 1 / \text { Waf1 }}(35), \mathrm{p} 15^{\text {INK }^{2}}(36,37), \mathrm{p} 16^{\text {INK4a }}$ (38), vimentin (50), fibronectin (51), PAI (52), and several SASP components (53, 54). Some of these upregulated gene expressions critically control the cell senescence itself, and contributes to the aging process and age-related diseases (55-59). Among the senescence-associated gene products, synthesis and secretion of SASP components have recently been of interest due to their potential link to various age-related diseases $(22,60-62)$.

\section{TYPES OF SENESCENCE-ASSOCIATED SECRETORY PHENOTYPE COMPONENTS}

The culture medium of senescent cells is enriched with secreted proteins $(63,64)$. The functional involvement of the secreted proteins in age-associated pathologies was initially recognized in a study by Campisi et al., where the secreted factors from senescent fibroblasts, especially MMP3, promoted the transformation of premalignant mammary epithelial cells $(65,66)$. This observation confirmed the belief that senescence might act as a tumor suppressor mechanism through the irreversible senescence arrest feature, thus emphasizing its potential to act as a double-edged sword within the tumor microenvironment. In addition, there is accumulating evidence that senescent cells secrete a variety of inflammatory cytokines, chemokines, proteases, and other immune modulators (67). As a result, it is predicted that senescent cells modify the tissue microenvironment in vivo by massive alteration of SASP expression (68). Most importantly, studies have revealed that the secretion of SASP components is conserved between human and mouse cells in vitro and in vivo (21, 22, 60-62), and it commonly occurs in the progress to senescence of various cell types, such as fibroblasts, epithelial cells, endothelial cells, and astrocytes $(22,69,70)$. Currently, some SASP components are used as general markers of senescence (60). These observations led us to investigate in detail the action mechanisms of SASP components in diverse age-related pathologies.

To thoroughly investigate the possible mechanisms by which SASP contributes to age-related pathologies, we have divided the SASP-related factors into three categories, depending on the mode of action that initiates the SASP activity: receptor-requiring SASP, direct-acting SASP, and SASP regulatory factors.

\section{Receptor-requiring SASP}

These factors include cytokines (interleukins), chemokines, and growth factors, which generally initiate their innate functions by binding to their respective receptors on the surface of target cells, and activating receptor-mediated intracellular signal transduction pathways. The most prominent SASP cytokines are interleukin (IL)- $6,-8$, and $-1 \alpha$; their mechanism of action in, and their contributions to cancer, are well understood. IL-6 secretion is markedly augmented in DNA damage and oncogene-induced senescence of mouse and human keratino- cytes, melanocytes, monocytes, fibroblasts, and epithelial cells $(22,61,71)$. Interestingly, IL-6 expression is upregulated by IL-1 $(72,73)$, which is also known to be overexpressed and secreted by senescent cells (74); this implies that the SASP component expression may be sequentially regulated in a hierarchal cascade through their autocrine activity. Among the chemokines, GRO $\alpha$ (CXCL-1), GRO $\beta$ (CXCL-2), MCP-1 (CCL-2), RANTES (CCL5), HCC-4 (CCL-16), eotaxin-3 (CCL-26), and MIP-3 $\alpha$ (CCL-20) are reported to be secreted by senescent cells $(21,60)$. They exert their biological effects by interacting with their own G protein-linked transmembrane receptors (chemokine receptors). Increased expression of many growth factors such as HGF, FGF, TGF $\beta 1$ and GM-CSF, has also been reported in various types of senescent cells $(21,61,75)$. The effect of these receptor-requiring SASP components on age-related diseases, including inflammatory diseases and cancer, relies on the target cells that express their respective receptors. The target cell may be the senescent cell itself, thereby aggravating or shielding the cell from senescence progression. Alternatively, the target cells may be various types of normal cells that are nearby; in this case, the cellular environment may be gradually modified to give rise to a pathological condition, or to create an environment in which multiple pathologies may arise.

\section{Direct-acting SASP}

Many matrix metalloproteinases (MMPs) belong to this group. MMP family members that are consistently upregulated in human and mouse fibroblasts undergoing replicative or stress-induced senescence include stromelysin-1 and -2 (also called MMP-3 and -10, respectively) and collagenase-1 (MMP-1) (66, 76-78). Another family of proteases that belongs to this SASP category are the serine proteases: urokinase-type or tissue-type plasminogen activators (UPA and tPA, respectively) $(24,67$, 79). These direct-acting SASP components exert their proteolytic activities on their respective substrates, mostly extracellular matrix $(E C M)$ proteins, the extracellular portion of membrane-anchoring proteins, or some soluble molecules released from cells, thereby modifying the extracellular microenvironment. Although non-protein small molecules such as ROS, transported ions, and metabolites may also belong to this group, they will not be discussed in this review since they are not proteins.

\section{SASP regulatory factors}

Members of this group include TIMPs, PAIs, and IGFBPs. These factors do not have their own enzymatic or signal triggering activities, but modulate the activities of receptor-requiring or direct-acting SASP components by binding to them. The TIMPs comprise of a family of four protease inhibitors: TIMP1, TIMP2, TIMP3, and TIMP4. TIMPs are generally known to inhibit most of the activated MMPs, but in some cases they are known to form complexes with the latent form of MMPs, such as MMP2 and MMP9 (80). The complex of TIMP2 with latent 
MMP2 (pro-MMP2) serves to facilitate the activation of proMMP2 at the cell surface by MT1-MMP (MMP14), a membrane-anchored MMP. The role of the pro-MMP9/TIMP1 complex has yet to be elucidated (80). TIMPs are known to be highly inducible in response to many cytokines and hormones (81), suggesting that their upregulated expression in senescent cells may be the result of the autocrine activity of SASP cytokines.

PAl-1 is a member of the serine protease inhibitor family (serpin); also known as SERPINE1, it functions as the major inhibitor of IPA and UPA, regulating fibrinolysis (the physiological breakdown of blood clots). PAI-1 also blocks the activity of MMPs, which play a crucial role in the invasion of malignant cells across the basal lamina (82). In addition, physiological roles for PAI-1 in metabolic diseases, such as insulin resistance and cardiovascular disease, have often been reported $(83,84)$. PAI-2 (SERPINB2) is primarily known to be secreted by the placenta. Recently, it has also been reported to bind to several intracellular and extracellular proteins, and to play a role in the regulation of adaptive immunity (85).

IGFBPs act as carrier proteins for insulin-like growth factor (IGF) and help to maintain the half-life of circulating IGFs in many tissues, thereby modulating IGF signaling depending on the tissue context $(86,87)$. In humans, IGFBPs are transcribed from seven genes, IGFBP1-7, which share a 50\% homology (88). Despite their similarities, the importance of IGF signaling in various pathologies, and the subtle structural differences among the IGFBPs, indicate their tremendous potential roles in modulating age-related diseases.

\section{STAGE-SPECIFIC EXPRESSION OF SASP COMPONENTS IN THE PROGRESS OF SENESCENCE}

Senescent cells express only selected SASP components at different stages of senescence progression. For example, IL-1 $\alpha$ expression is one of the earliest events after senescent growth arrest, and its autocrine activity induces several second-wave SASP components, including IL- 6 and -8 , via activation of nuclear factor $\kappa \mathrm{B}(\mathrm{NF}-\mathrm{\kappa B})$ and $\mathrm{C} / \mathrm{EBP} \beta$ (89). In cultures, our recent time-series of gene expression profiles during the progression of replicative senescence in human diploid fibroblasts (HDF) supports the idea of stage-specific SASP expression (90). These observations imply that senescent cells at different stages may have varying impacts on the progress of even the same age-re-

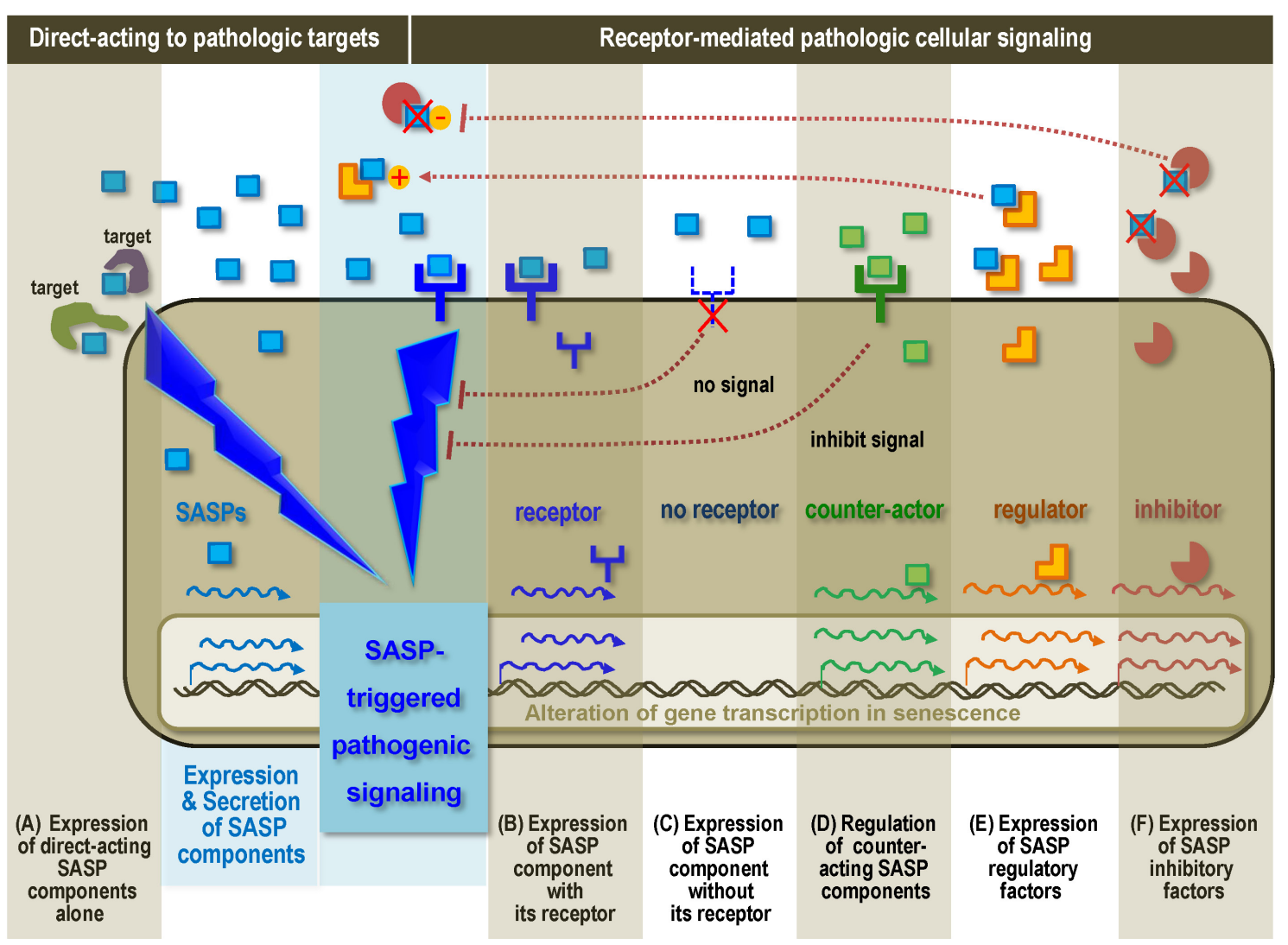

Fig. 1. Possible action mechanisms of SASPs, based on differential expression of SASP-related factors. 
lated disease, such as cancer.

\section{POSSIBLE ACTION MECHANISMS OF SASP BY DIFFERENTIAL EXPRESSION OF SASP-RELATED FACTORS}

As described above, the overall contribution of individual SASP components to the progress of senescence itself or to age-related pathologies does not depend on the expression levels of the SASP component alone; it is also affected by the inhibitory, regulatory, or counteracting SASP molecules coexpressed by the same senescent cell. In addition, expression levels of SASP component receptors on the target cell, which may be the senescent cell itself or nearby cells, are another critical factor. Based on our previous time series of gene expression profiles for all of the SASP-related factors, including SASP components, SASP regulators, and SASP receptors (90), the possible action mechanisms of SASP components can be placed into six groups (Fig. 1).

\section{Expression of direct-acting SASP components alone}

Direct-acting SASP proteases (proteinases) can modulate the tissue microenvironment by regulating turnover of the ECM through processing of ECM factors, including collagen, fibronectin, and proteoglycans (Fig. 1A). By ectodomain shedding, they can also regulate the activity of certain growth factors and cytokines such as HB-EGF and TNF- $\alpha$, and membrane-spanning receptors such as c-Met (91-93).

\section{Expression of SASP component with its receptor}

Senescent cells express a diverse array of receptor-requiring SASP factors. The effect and action mechanisms of these factors can be very different, depending on the cell types expressing their receptors. If a senescent cell expresses both a SASP component and its receptor, then the SASP component will signal to the senescent cell itself via autocrine activity (Fig. 1B). A good example is the increased expression during senescence of both IL-8 and its receptor, IL-8RB (CXCR2), and their involvement in mediating senescence $(90,94-96)$. Similar expression patterns for IL-8 and IL-8RB were also observed in our study of replicative senescence in HDF (Table 1).

\section{Expression of SASP component without its receptor}

If senescent cells express only a SASP component while suppressing expression of its receptor, then the SASP component will affect nearby nonsenescent cells or other target cells expressing its receptor via paracrine activity (Fig. 1C). Similar expression profiles for this group are seen for IL-17 and -20 (90), but their functional relevance to senescence or age-associated diseases has yet to be elucidated.

\section{Regulation of counteracting SASP components}

Senescent cells also regulate counteracting SASP components $\left(\mathrm{SASP}_{\mathrm{C}}\right)$ that block specific SASP-triggered signaling events (Fig. 1D). Anti-inflammatory cytokines such as IL-4, -10, -13, and -35 may act as SASPc. IL-10 and -13 expression was found to be downregulated during replicative senescence of HDF (90), implying that proinflammatory signaling can be activated in senescence.

\section{Expression of SASP regulatory factors}

Senescent cells express regulatory SASP components $\left(S_{A S P}\right)$ that prolong or promote certain SASP-mediated signaling events by binding to their respective receptors or to the SASP component itself (Fig. 1E). Representative examples are the IGFBPs, which are IGF signaling regulators. Insulin/IGF-mediated signaling is known to be a significant contributor to biological aging in many organisms $(14,97)$. Therefore, it is not surprising that the IGFBPs would have a role in senescence and aging. Increased expression of IGFBPs such as IGFBP2, IGFBP5, and IGFBP7, and their involvement in cell senescence, organismal aging, and age-associated diseases have been reported recently $(1,4,98)$. By contrast, IGFBP6 expression is associated with delayed replicative senescence of HDF (99). Our previous study of gene expression profiles during replicative senescence also demonstrated that IGFBP4 and IGFBP6 were downregulated, whereas IGFBP2, IGFBP3, IGFBP5, and IGFBP7 were upregulated (90), supporting their different contributions to senescence and the aging progress.

\section{Expression of SASP inhibitory factors}

The release of inhibitory SASP components $\left(\mathrm{SASP}_{\mathrm{I}}\right)$ inhibits SASP component activity by direct interactions (Fig. 1F). PAI

Table 1. Combined activity of interleukins and expression of their respective receptors*

\begin{tabular}{|c|c|c|c|c|}
\hline \multirow{2}{*}{ Names } & \multicolumn{2}{|c|}{ Expression changes during senescence } & \multicolumn{2}{|c|}{ Differential action mechanisms } \\
\hline & Interleukins & Receptors & Young cell & Senescent cell \\
\hline IL1A, IL1B, IL12 IL17, IL20 & Up & Down & High sensitivity & Paracrine action only \\
\hline IL6, IL8, IL21 & Up & Up & Suppressed overall action & Para- \& autocrine action \\
\hline IL13, IL15, IL18 & Down & Up & Paracrine action only & High sensitivity \\
\hline IL3, IL10, IL17 & Down & Down & Para- \& autocrine action & Loss of overall activity \\
\hline
\end{tabular}

*This table was created by reanalyzing previously reported results (90). 
and TIMP are good examples. Comi et al. proposed that PAI-1 could be used as a senescence marker for endothelial cells (15). However, another study reported that UPA and PAI- 1 are constitutively expressed in young, mitotically active cells, whereas tPA and PAI-2 are markedly increased in senescent lung epithelial cells, thus underlining the importance of senescenceassociated alterations in IPA activator activity (13). In addition, both MMPs and their inhibitors (TIMPs) are altered during replicative senescence (50). These alterations were further confirmed by the results of our previous study on replicative senescence (Table 2), suggesting that the senescence-associated activities of MMPs and PAs are not just due to the levels of the component itself, but also due to the balance between expression of SASP components and their respective SASP.

The above observations and the deduced mechanisms indicate that it is the well-orchestrated activity of all of the SASP components, rather than the activity of a single SASP component, that determines whether a senescent cell will be the source of specific age-related pathologies. Alternatively, this activity may modify the tissue microenvironment so that it is susceptible to age-associated pathologies.

\section{POTENTIAL ROLES OF SENESCENT CELLS IN AGE-ASSOCIATED PATHOLOGIES}

Although the molecular mechanisms of the individual SASP components involved in aging and age-associated diseases have been demonstrated, it is still undoubtedly complex and unclear as to how the senescent cells expressing multiple SASP-related factors contribute to these pathologies. When only the direct-acting SASP components are considered, the contribution of senescent cells to pathology would seem predictable, since the direct-acting SASP components mainly modify the surrounding tissue microenvironment by modulating the ECM. However, their ultimate activities still depend on the levels of coexpressed SASP ${ }_{I}$ and $\mathrm{SASP}_{\mathrm{R}}$. On the other hand, understanding the role of senescent cells in conjunction with the expression of receptor-requiring SASP components is obviously more difficult. Therefore, we propose below four classes of potential roles for senescent cells in age-associated pathologies. We have used the differential expression profiles of inter- leukins and their receptors as examples (Table 1 and Fig. 2).

Class I: senescent cells act as effector cells to modulate the microenvironment by releasing SASP components alone In this class, senescent cells express increased levels of certain SASP components but decreased levels of their respective receptors, whereas young cells express only the receptor. Thus, SASP components released from senescent cells target the young cells or other disease-prone cells, such as cancer cells, which express the SASP receptors, thereby generating a onesided paracrine communication from the senescent to the young (target) cell. In this scenario, senescent cells act as effector cells by attacking and modulating the surrounding nonsenescent cells via release of certain SASP components, eventually contributing to pathogenesis. Based on our previous results (Table 1 ), IL-1, $-2,-12,-17 d$, and -20 may act in this class.

\section{Class II: senescent cells communicate among themselves by coexpressing SASP components and their respective receptors}

When senescent cells, but not the surrounding nonsenescent cells, express both pathogenic SASP components and their receptors, SASP-mediated communication will occur only among senescent cells via both autocrine and paracrine activities. In this scenario, SASP may promote senescence progression or modify senescent cells to acquire pathogenic features (56). If the surrounding nonsenescent disease-prone cells, such as cancer cells, express the receptor, then paracrine communication from the senescent cell to the disease-prone cell may promote pathogenesis. This concept is supported by several recent reports which show that IL- 6 and -8 released from senescent cells promote the epithelial-mesenchymal transition and invasiveness of premalignant epithelial cells in culture (51). Representative SASP components in this class may include IL-6, $-8,-21$, and -32 (Table 1 and Fig. 2).

\section{Class III: senescent cells act as target cells expressing SASP receptors only}

In some cases, senescent cells express high levels of certain SASP receptors alone, without expressing their respective SASP components, whereas nearby young cells express the SASP

Table 2. Combined activity of MMPs and their inhibitory factors*

\begin{tabular}{|c|c|c|c|}
\hline \multirow{2}{*}{\multicolumn{2}{|c|}{ Expression changes during senescence }} & \multicolumn{2}{|c|}{ Possible action mechanisms } \\
\hline & & \multirow{2}{*}{$\begin{array}{c}\text { Young cell } \\
\text { Combined activity of MMPs } \\
\text { (MMP2, MMP11, MMP20, } \\
\text { MMP27) and TIMP4 }\end{array}$} & \multirow{2}{*}{$\begin{array}{c}\text { Senescent cell } \\
\text { Combined activity of MMPs } \\
\text { (MMP1, MMP3, MMP12) and } \\
\text { TIMPs (TIMP1, TIMP2, TIMP3) }\end{array}$} \\
\hline $\begin{array}{l}\text { MMP1, MMP3, MMP12 } \\
\text { MMP2, MMP11, MMP20, MMP27 } \\
\text { TIMP1, TIMP2, TIMP3 } \\
\text { TIMP4 }\end{array}$ & $\begin{array}{l}\text { Up } \\
\text { Down } \\
\text { Up } \\
\text { Down }\end{array}$ & & \\
\hline
\end{tabular}

*This table was created by reanalyzing previously reported results (90). 


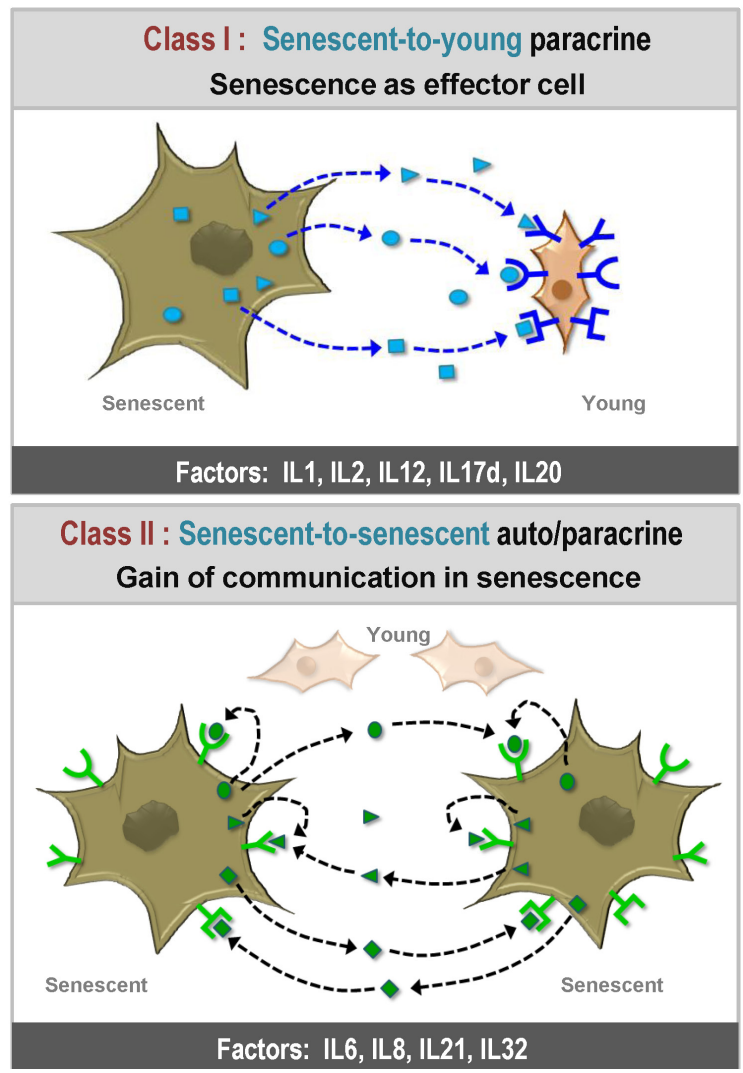

Fig. 2. Potential roles of senescent cells in age-associated pathologies.

components. In this scenario, the senescent cell becomes susceptible to these SASP components, probably making itself a target cell by a one-sided paracrine communication from the proliferating young cell to the senescent cell. This class may include IL-7, -13, -15, -18, -19, -23, -25, and -34 (Table 1 and Fig. 2). However, it is unclear whether these SASP components act to promote senescence or to destine the senescent cell to become a source of pathogenesis.

Class IV: senescent cells lose cell-to-cell communication by suppressing expression of SASP components and their respective receptors

If senescent cells suppress the expression of specific SASP components and their receptors, they lose their autocrine and paracrine communication. IL-3, -10,-17a, $-17 \mathrm{~b}$, and $-17 \mathrm{c}$ are in this class (Table 1 and Fig. 2). Interestingly, IL-10 is known to act as an anti-inflammatory cytokine and has a protective role against atherosclerosis (55). Therefore, losing IL-10-mediated communication may accelerate other inflammatory responses and result in pathogenic progression.

We have proposed four possible mechanistic contributions of senescent cells to age-associated pathologies by referring

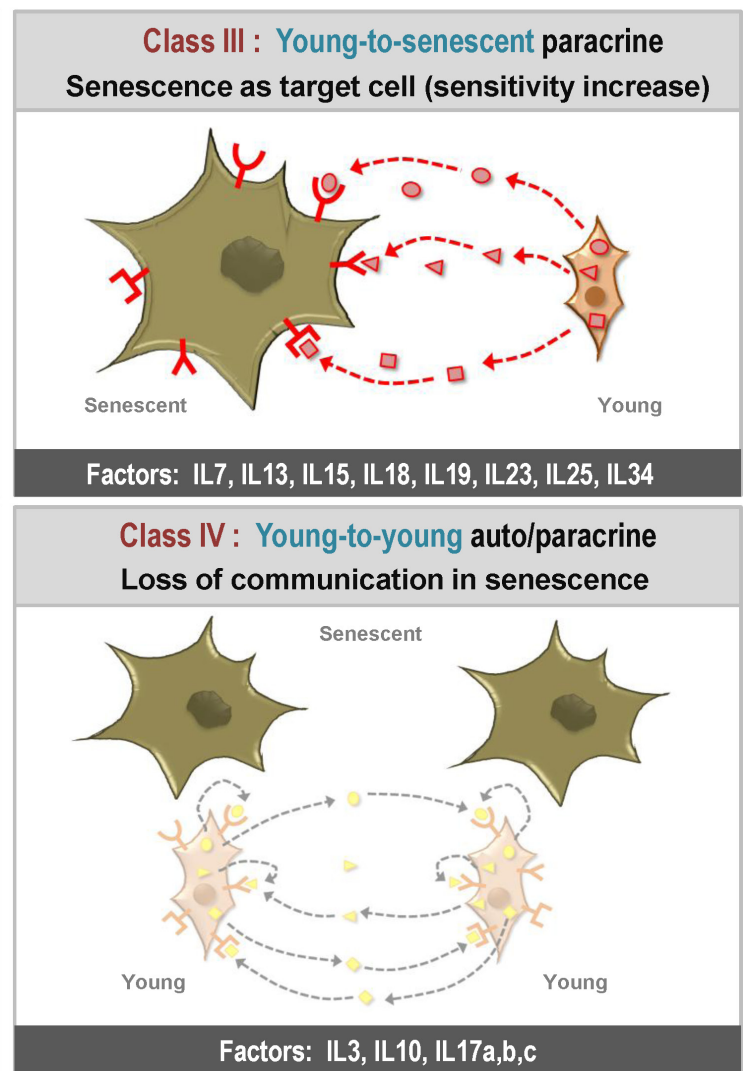

only to the differential expression of interleukins and their receptors. However, all four of these scenarios may occur at the same time in the same tissue, suggesting that their orchestrated actions are involved in promoting senescence-associated pathogenic tissue environments and remodeling. Moreover, considering all of the contributions made by the other SASP-related factors, it is challenging to explain the roles of senescence in pathogenesis. Nevertheless, elucidating SASP-mediated extracellular microenviromental remodeling, in addition to senescence-related intracellular signaling, is crucial in establishing a correct and complete understanding of senescence and its related pathologies. More detailed studies of the integrity of the SASP-related factors, in terms of the components and their functional interconnections, are needed to develop and exploit appropriate prosenescent or antisenescent therapeutic strategies.

\section{ACKNOWLEDGEMENTS}

This work was supported by the National Research Foundation of Korea (NRF) grant funded by the Korea government (MSIP) (2012R1A5A2048183). 


\section{REFERENCES}

1. Chen D, Yoo BK, Santhekadur PK et al (2011) Insulin-like growth factor-binding protein-7 functions as a potential tumor suppressor in hepatocellular carcinoma. Clin Cancer Res 17, 6693-6701

2. Jones AW, Yao Z, Vicencio JM, Karkucinska-Wieckowska A and Szabadkai G (2012) PGC-1 family coactivators and cell fate: roles in cancer, neurodegeneration, cardiovascular disease and retrograde mitochondria-nucleus signalling. Mitochondrion 12, 86-99

3. Chatrchyan S, Khachatryan V, Sirunyan AM et al (2011) Measurement of the inclusive jet cross section in pp collisions at radicals $=7 \mathrm{TeV}$. Phys Rev Lett 107, 132001

4. Sunderic M, Mihailovic N and Nedic O (2014) Protein molecular forms of insulin-like growth factor binding protein-2 change with aging. Exp Gerontol 58, 154-158

5. Minamino T, Miyauchi $H$, Yoshida T, Ishida $Y$, Yoshida $H$ and Komuro I (2002) Endothelial cell senescence in human atherosclerosis: role of telomere in endothelial dysfunction. Circulation 105, 1541-1544

6. Youdim MB and Riederer P (1993) The role of iron in senescence of dopaminergic neurons in Parkinson's disease. J Neural Transm Suppl 40, 57-67

7. Hengstler JG and Bolt HM (2008) Oxidative stress: from modification of cell-cycle related events, secondary messenger function, dysregulation of small GTPases, protein kinases and phosphatases to redox-sensitive cancer models. Arch Toxicol 82, 271-272

8. Jones RM and Neef N (2012) Interpretation and prediction of inhaled drug particle accumulation in the lung and its associated toxicity. Xenobiotica 42, 86-93

9. Hung WY, Huang KH, Wu CW et al (2012) Mitochondrial dysfunction promotes cell migration via reactive oxygen species-enhanced beta5-integrin expression in human gastric cancer SC-M1 cells. Biochim Biophys Acta 1820, $1102-1110$

10. Hoffmeister A, Ropolo A, Vasseur S et al (2002) The HMG-I/Y-related protein p8 binds to p300 and Pax2 transactivation domain-interacting protein to regulate the transactivation activity of the Pax2A and Pax2B transcription factors on the glucagon gene promoter. J Biol Chem 277, 22314-22319

11. Rohman L and Jones A (2012) Chilaiditi's sign. Emerg Med J 29, 86

12. Engelberg B and Jones B (2012) Exploring minimally invasive options: managing demands, expectations, and outcomes. Dent Today 31, 86, 88, 90 passim

13. West MD, Shay JW, Wright WE and Linskens MH (1996) Altered expression of plasminogen activator and plasminogen activator inhibitor during cellular senescence. Exp Gerontol 31, 175-193

14. Lapierre LR and Hansen M (2012) Lessons from C. elegans: signaling pathways for longevity. Trends Endocrinol Metab 23, 637-644

15. Comi P, Chiaramonte R and Maier JA (1995) Senescencedependent regulation of type 1 plasminogen activator inhibitor in human vascular endothelial cells. Exp Cell Res 219, 304-308

16. Sabharwal SS and Schumacker PT (2014) Mitochondrial
ROS in cancer: initiators, amplifiers or an Achilles' heel? Nat Rev Cancer 14, 709-721

17. Ballinger SW (2013) Beyond retrograde and anterograde signalling: mitochondrial-nuclear interactions as a means for evolutionary adaptation and contemporary disease susceptibility. Biochem Soc Trans 41, 111-117

18. Dimri GP, Lee X, Basile G et al (1995) A biomarker that identifies senescent human cells in culture and in aging skin in vivo. Proc Natl Acad Sci U S A 92, 9363-9367

19. Bateman A and Bennett HP (2009) The granulin gene family: from cancer to dementia. Bioessays 31, 1245-1254

20. Beckman KB and Ames BN (1999) Endogenous oxidative damage of mtDNA. Mutat Res 424, 51-58

21. Biswas G, Adebanjo OA, Freedman BD et al (1999) Retrograde $\mathrm{Ca} 2+$ signaling in $\mathrm{C} 2 \mathrm{C} 12$ skeletal myocytes in response to mitochondrial genetic and metabolic stress: a novel mode of inter-organelle crosstalk. EMBO J 18, 522533

22. Biswas G, Anandatheerthavarada HK, Zaidi M and Avadhani NG (2003) Mitochondria to nucleus stress signaling: a distinctive mechanism of NFkappaB/Rel activation through calcineurin-mediated inactivation of IkappaBbeta. J Cell Biol 161, 507-519

23. Berneburg $M$, Kamenisch $Y$, Krutmann J and Rocken $M$ (2006) 'To repair or not to repair - no longer a question': repair of mitochondrial DNA shielding against age and cancer. Exp Dermatol 15, 1005-1015

24. He X, Zhou A, Lu H et al (2013) Suppression of mitochondrial complex I influences cell metastatic properties. PLoS One 8, e61677

25. Boiteux S and Radicella JP (2000) The human OGG1 gene: structure, functions, and its implication in the process of carcinogenesis. Arch Biochem Biophys 377, 1-8

26. Brandon M, Baldi P and Wallace DC (2006) Mitochondrial mutations in cancer. Oncogene 25, 4647-4662

27. Zhang YQ and Herman B (2008) Expression and modification of ARC (apoptosis repressor with a CARD domain) is distinctly regulated by oxidative stress in cancer cells. J Cell Biochem 104, 818-825

28. Bogenhagen DF, Pinz KG and Perez-Jannotti RM (2001) Enzymology of mitochondrial base excision repair. Prog Nucleic Acid Res Mol Biol 68, 257-271

29. Guettouche T, Boellmann F, Lane WS and Voellmy R (2005) Analysis of phosphorylation of human heat shock factor 1 in cells experiencing a stress. BMC Biochem 6, 4

30. Butow RA and Avadhani NG (2004) Mitochondrial signaling: the retrograde response. Mol Cell 14, 1-15

31. Byun HO, Jung HJ, Seo YH et al (2012) GSK3 inactivation is involved in mitochondrial complex IV defect in transforming growth factor (TGF) beta1-induced senescence. Exp Cell Res 318, 1808-1819

32. Amuthan G, Biswas G, Ananadatheerthavarada HK, Vijayasarathy C, Shephard HM and Avadhani NG (2002) Mitochondrial stress-induced calcium signaling, phenotypic changes and invasive behavior in human lung carcinoma A549 cells. Oncogene 21, 7839-7849

33. Byun HO, Kim HY, Lim JJ, Seo YH and Yoon G (2008) Mitochondrial dysfunction by complex II inhibition delays overall cell cycle progression via reactive oxygen species production. J Cell Biochem 104, 1747-1759 
34. Campagnoni CW, Landry CF, Pribyl TM et al (2001) Identification of genes in the oligodendrocyte lineage through the analysis of conditionally immortalized cell lines. Dev Neurosci 23, 452-463

35. Cano CE, Hamidi T, Sandi MJ and lovanna JL (2011) Nupr1: the Swiss-knife of cancer. J Cell Physiol 226, 1439-1443

36. Carew JS and Huang P (2002) Mitochondrial defects in cancer. Mol Cancer 1, 9

37. Chae S, Ahn BY, Byun K et al (2013) A systems approach for decoding mitochondrial retrograde signaling pathways. Sci Signal 6, rs4

38. Chang CJ, Yin PH, Yang DM et al (2009) Mitochondrial dysfunction-induced amphiregulin upregulation mediates chemo-resistance and cell migration in HepG2 cells. Cell Mol Life Sci 66, 1755-1765

39. Chatterjee A, Mambo E and Sidransky D (2006) Mitochondrial DNA mutations in human cancer. Oncogene 25, 4663-4674

40. Stein GH and Dulic V (1998) Molecular mechanisms for the senescent cell cycle arrest. J Investig Dermatol Symp Proc 3, 14-18

41. Hwang ES, Yoon G and Kang HT (2009) A comparative analysis of the cell biology of senescence and aging. Cell Mol Life Sci 66, 2503-2524

42. Cheung ST, Wong SY, Leung KL et al (2004) Granulin-epithelin precursor overexpression promotes growth and invasion of hepatocellular carcinoma. Clin Cancer Res 10, 7629-7636

43. Chowdhury UR, Samant RS, Fodstad O and Shevde LA (2009) Emerging role of nuclear protein 1 (NUPR1) in cancer biology. Cancer Metastasis Rev 28, 225-232

44. Clayton DA (1998) Nuclear-mitochondrial intergenomic communication. Biofactors 7, 203-205

45. Cortopassi G and Wang E (1995) Modelling the effects of age-related mtDNA mutation accumulation; complex I deficiency, superoxide and cell death. Biochim Biophys Acta $1271,171-176$

46. Kim YM, Shin HT, Seo YH et al (2010) Sterol regulatory element-binding protein (SREBP)-1-mediated lipogenesis is involved in cell senescence. J Biol Chem 285, 2906929077

47. Khachatryan V, Sirunyan AM, Tumasyan A et al (2011) Measurement of the $\mathrm{B}(+)$ production cross section in $\mathrm{pp}$ collisions at sqrt[s] = 7 TeV. Phys Rev Lett 106, 112001

48. Czarnecka AM, Kukwa W, Krawczyk T, Scinska A, Kukwa A and Cappello F (2010) Mitochondrial DNA mutations in cancer-from bench to bedside. Front Biosci 15, 437-460

49. Seo M and Lee YH (2014) PFKFB3 regulates oxidative stress homeostasis via its S-glutathionylation in cancer. J Mol Biol 426, 830-842

50. Millis AJ, Hoyle M, McCue HM and Martini H (1992) Differential expression of metalloproteinase and tissue inhibitor of metalloproteinase genes in aged human fibroblasts. Exp Cell Res 201, 373-379

51. Coppe JP, Patil CK, Rodier F et al (2008) Senescence-associated secretory phenotypes reveal cell-nonautonomous functions of oncogenic RAS and the p53 tumor suppressor. PLoS Biol 6, 2853-2868

52. Imamura H, Matsuyama Y, Tanaka E et al (2003) Risk fac- tors contributing to early and late phase intrahepatic recurrence of hepatocellular carcinoma after hepatectomy. J Hepatol 38, 200-207

53. Rodier F and Campisi J (2011) Four faces of cellular senescence. J Cell Biol 192, 547-556

54. Vital P, Castro P, Tsang S and Ittmann M (2014) The senescence-associated secretory phenotype promotes benign prostatic hyperplasia. Am J Pathol 184, 721-731

55. Han X and Boisvert WA (2015) Interleukin-10 protects against atherosclerosis by modulating multiple atherogenic macrophage function. Thromb Haemost 113, 505-512

56. Groppo R and Richter JD (2011) CPEB control of NF-kappaB nuclear localization and interleukin-6 production mediates cellular senescence. Mol Cell Biol 31, 2707-2714

57. Kulju KS and Lehman JM (1995) Increased p53 protein associated with aging in human diploid fibroblasts. Exp Cell Res 217, 336-345

58. Salminen A and Kaarniranta K (2010) Glycolysis links p53 function with NF-kappaB signaling: impact on cancer and aging process. J Cell Physiol 224, 1-6

59. Brookes S, Rowe J, Gutierrez Del Arroyo A, Bond J and Peters G (2004) Contribution of p16 (INK4a) to replicative senescence of human fibroblasts. Exp Cell Res 298, 549559

60. Delsite R, Kachhap S, Anbazhagan R, Gabrielson E and Singh KK (2002) Nuclear genes involved in mitochondria-to-nucleus communication in breast cancer cells. Mol Cancer 1,6

61. Demorrow S (2013) Progranulin: a novel regulator of gastrointestinal cancer progression. Transl Gastrointest Cancer $2,145-151$

62. Di Bella D, Lazzaro F, Brusco A et al (2010) Mutations in the mitochondrial protease gene AFG3L2 cause dominant hereditary ataxia SCA28. Nat Genet 42, 313-321

63. Dzierzbicki P, Koprowski P, Fikus MU, Malc E and Ciesla Z (2004) Repair of oxidative damage in mitochondrial DNA of Saccharomyces cerevisiae: involvement of the MSH1-dependent pathway. DNA Repair (Amst) 3, 403-411

64. DiMauro S and Schon EA (2003) Mitochondrial respiratory-chain diseases. N Engl J Med 348, 2656-2668

65. Encinar JA, Mallo GV, Mizyrycki C et al (2001) Human p8 is a $\mathrm{HMG}-1 /$-like protein with DNA binding activity enhanced by phosphorylation. J Biol Chem 276, 2742-2751

66. Finley LW and Haigis MC (2009) The coordination of nuclear and mitochondrial communication during aging and calorie restriction. Ageing Res Rev 8, 173-188

67. Ashok BT, Ahmad J and Ali R (1998) Immunochemical detection of oxidative DNA damage in cancer and aging using anti-reactive oxygen species modified DNA monoclonal antibody. Int J Biochem Cell Biol 30, 1367-1377

68. Gardiner K (1995) Human genome organization. Curr Opin Genet Dev 5, 315-322

69. Goruppi S, Bonventre JV and Kyriakis JM (2002) Signaling pathways and late-onset gene induction associated with renal mesangial cell hypertrophy. EMBO J 21, 5427-5436

70. Giroux V, Malicet C, Barthet $M$ et al (2006) p8 is a new target of gemcitabine in pancreatic cancer cells. Clin Cancer Res 12, 235-241

71. Goruppi S and Kyriakis JM (2004) The pro-hypertrophic 
basic helix-loop-helix protein p8 is degraded by the ubiquitin/proteasome system in a protein kinase B/Akt- and glycogen synthase kinase-3-dependent manner, whereas endothelin induction of $\mathrm{p} 8 \mathrm{mRNA}$ and renal mesangial cell hypertrophy require NFAT4. J Biol Chem 279, 2095020958

72. Gredilla R, Garm C and Stevnsner T (2012) Nuclear and mitochondrial DNA repair in selected eukaryotic aging model systems. Oxid Med Cell Longev 2012, 282438

73. Grollman AP and Moriya M (1993) Mutagenesis by 8-oxoguanine: an enemy within. Trends Genet 9, 246-249

74. Guha M and Avadhani NG (2013) Mitochondrial retrograde signaling at the crossroads of tumor bioenergetics, genetics and epigenetics. Mitochondrion 13, 577-591

75. Takahashi T, Yoshida H, Mamada Y, Taniai $N$ and Tajiri $T$ (2009) Balloon-occluded retrograde transvenous obliteration for gastric varices in a child with extrahepatic portal venous obstruction. J Nippon Med Sch 76, 173-178

76. Hamidi T, Algul H, Cano CE et al (2012) Nuclear protein 1 promotes pancreatic cancer development and protects cells from stress by inhibiting apoptosis. J Clin Invest 122, 2092-2103

77. Hamidi T, Cano CE, Grasso D et al (2012) Nupr1-aurora kinase A pathway provides protection against metabolic stress-mediated autophagic-associated cell death. Clin Cancer Res 18, 5234-5246

78. Haque A, Nishikawa M, Qian W et al (2006) Lack of mitochondrial DNA enhances growth of hepatocellular carcinoma in vitro and in vivo. Hepatol Res 36, 209-216

79. Hashiguchi K, Stuart JA, de Souza-Pinto NC and Bohr VA (2004) The C-terminal alphaO helix of human Ogg1 is essential for 8-oxoguanine DNA glycosylase activity: the mitochondrial beta-Ogg1 lacks this domain and does not have glycosylase activity. Nucleic Acids Res 32, 55965608

80. Brew K, Dinakarpandian D and Nagase H (2000) Tissue inhibitors of metalloproteinases: evolution, structure and function. Biochim Biophys Acta 1477, 267-283

81. Reichenstein M, Reich R, LeHoux JG and Hanukoglu I (2004) ACTH induces TIMP-1 expression and inhibits collagenase in adrenal cortex cells. Mol Cell Endocrinol 215, 109-114

82. Binder BR, Christ G, Gruber F et al (2002) Plasminogen activator inhibitor 1: physiological and pathophysiological roles. News Physiol Sci 17, 56-61

83. Alessi MC, Poggi M and Juhan-Vague I (2007) Plasminogen activator inhibitor- 1 , adipose tissue and insulin resistance. Curr Opin Lipidol 18, 240-245

84. Gils A and Declerck PJ (2004) The structural basis for the pathophysiological relevance of PAI-I in cardiovascular diseases and the development of potential PAI-I inhibitors. Thromb Haemost 91, 425-437
85. Schroder WA, Major $L$ and Suhrbier A (2011) The role of SerpinB2 in immunity. Crit Rev Immunol 31, 15-30

86. Hwa V, Oh Y and Rosenfeld RG (1999) The insulin-like growth factor-binding protein (IGFBP) superfamily. Endocr Rev 20, 761-787

87. Stewart CE, Bates PC, Calder TA, Woodall SM and Pell JM (1993) Potentiation of insulin-like growth factor-I (IGF-I) activity by an antibody: supportive evidence for enhancement of IGF-I bioavailability in vivo by IGF binding proteins. Endocrinology 133, 1462-1465

88. Clemmons DR, Busby WH, Arai T et al (1995) Role of insulin-like growth factor binding proteins in the control of IGF actions. Prog Growth Factor Res 6, 357-366

89. Orjalo AV, Bhaumik D, Gengler BK, Scott GK and Campisi $\mathrm{J}$ (2009) Cell surface-bound IL-1alpha is an upstream regulator of the senescence-associated IL-6/IL-8 cytokine network. Proc Natl Acad Sci U S A 106, 17031-17036

90. Kim YM, Byun HO, Jee BA et al (2013) Implications of time-series gene expression profiles of replicative senescence. Aging Cell 12, 622-634

91. Chow FL and Fernandez-Patron C (2007) Many membrane proteins undergo ectodomain shedding by proteolytic cleavage. Does one sheddase do the job on all of these proteins? IUBMB Life 59, 44-47

92. Hayashida K, Bartlett AH, Chen Y and Park PW (2010) Molecular and cellular mechanisms of ectodomain shedding. Anat Rec (Hoboken) 293, 925-937

93. Athauda G, Giubellino A, Coleman JA et al (2006) c-Met ectodomain shedding rate correlates with malignant potential. Clin Cancer Res 12, 4154-4162

94. Acosta JC and Gil J (2009) A role for CXCR2 in senescence, but what about in cancer? Cancer Res 69, 21672170

95. Freund A, Orjalo AV, Desprez PY and Campisi J (2010) Inflammatory networks during cellular senescence: causes and consequences. Trends Mol Med 16, 238-246

96. Guo H, Liu Z, Xu B et al (2013) Chemokine receptor CXCR2 is transactivated by p53 and induces p38-mediated cellular senescence in response to DNA damage. Aging Cell 12, 1110-1121

97. Kenyon C, Chang J, Gensch E, Rudner A and Tabtiang R (1993) A C. elegans mutant that lives twice as long as wild type. Nature 366, 461-464

98. Elzi DJ, Lai Y, Song M, Hakala K, Weintraub ST and Shiio $Y$ (2012) Plasminogen activator inhibitor 1-insulin-like growth factor binding protein 3 cascade regulates stressinduced senescence. Proc Natl Acad Sci U S A 109, 12052-12057

99. Micutkova L, Diener T, Li C et al (2011) Insulin-like growth factor binding protein-6 delays replicative senescence of human fibroblasts. Mech Ageing Dev 132, 468-479 